\title{
Improving communication, teamwork, and action during a "cannot intubate cannot oxygenate (CICO)" emergency: employing CICO as a cognitive aid mnemonic
}

\author{
Laura V. Duggan, MD, FRCPC · Peter G. Brindley, MD, FRCPC · J. Adam Law, MD, FRCPC
}

Received: 9 July 2018/Revised: 12 July 2018/Accepted: 12 July 2018/Published online: 14 August 2018

(c) Canadian Anesthesiologists' Society 2018

Anesthesiology, critical care, and emergency medicine share the common goal of safe airway management. While often performed uneventfully, adverse patient anatomy, physiology, or situational acuity can precipitate an airway emergency. The outcome of an airway emergency may result as much from non-technical skills as from technical expertise. $^{1-5}$ Airway guidelines now highlight the importance of these non-technical skills such as teamwork and effective communication during airway emergencies. ${ }^{6,7}$

One example of an airway emergency requiring timely and structured teamwork is the rare life-threatening "cannot intubate, cannot oxygenate" (CICO) emergency. The CICO emergency requires immediate transition from tracheal intubation attempts to an emergency front-of-neck airway (eFONA) procedure. ${ }^{6, \mathrm{~A}}$ While often technically successful, eFONA is frequently performed too late to save the patient from hypoxemic brain damage or death. ${ }^{1,8}$ This delay may reflect the lack of a vital non-technical skill-i.e., establishing a shared mental model about what is occurring and initiating the predictable next steps required to remedy the CICO emergency. ${ }^{9}$ Lack of such a shared mental model may be related to: i) the failure to recognize a CICO emergency; ii) once recognized, the failure to promptly and

L. V. Duggan, MD, FRCPC ( $\varangle)$

Department of Anesthesiology, Pharmacology and Therapeutics, University of British Columbia, Vancouver, BC, Canada

e-mail: lauravduggan@gmail.com

P. G. Brindley, MD, FRCPC

Department of Critical Care Medicine, University of Alberta, Edmonton, AB, Canada

J. A. Law, MD, FRCPC

Department of Anesthesia, Pain Management and Perioperative Medicine, Dalhousie University, Halifax, NS, Canada unequivocally declare a CICO emergency to the entire team; and/or iii) once a CICO emergency is declared, the failure to rapidly perform eFONA. Cognitive aids, like checklists, appear to be more effective if they direct action rather than simply listing concerns. ${ }^{10}$ This article offers a simple mnemonic as a four-part cognitive aid-corresponding to the letters "CICO" - that can be easily recalled and summarizes the key sequential steps to identifying and managing the CICO airway emergency.

\section{C: call it and communicate it}

CICO is defined by the inability to tracheally intubate a patient (even after only a single attempt) combined with the failure to maintain oxygenation using both face mask and supraglottic airway (SGA) device ventilation. Delayed recognition of an evolving CICO emergency is multifactorial but often involves inadequate or deteriorating situational awareness. ${ }^{9}$ Situational awareness involves obtaining the information (e.g., "I cannot tracheally intubate the patient and cannot maintain oxygen saturation despite optimized attempts at ventilation using both face mask and SGA"), then understanding the information (e.g., "this is a CICO emergency and the need for eFONA is imminent"), and finally correctly acting on the information (e.g., "further attempts at tracheal intubation are inappropriate-eFONA must occur without delay").

Failure to obtain or understand the information that a CICO emergency is occurring may follow from its developing over time rather than suddenly-i.e., so-called

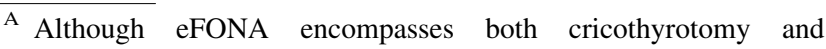
tracheostomy procedures, in this article it refers specifically to cricothyrotomy.
} 


\title{
Cannot Intubate, Cannot Oxygenate (CICO) Checklist Mnemonic
}

\author{
C Call it and communicate it: \\ - Communicate explicitly, in an assertively graded manner: from "is this a CICO airway?" to "this \\ is a CICO airway" \\ - $\quad$ Call for help. Ask help received to summarize the situation they observe. \\ I Incision is Imminent: \\ - $\quad$ Get equipment: a bougie, a 6.0 endotracheal tube and a \#10 or \#20 scalpel; \\ - $\quad$ Paralyze the patient, make final attempts at face mask and supraglottic airway oxygenation \\ while equipment is obtained and opened;

\section{Cut vertically:} \\ - Do not wait for surgical personnel to arrive. \\ - Without delay, cut 4-6 cm vertically with the scalpel blade in the midline. Extend if required. \\ - $\quad$ Palpate the cricothyroid membrane after vertical incision is made. \\ O Over-a-bougie technique: \\ - Identify the cricothyroid membrane by palpation within the wound after skin and \\ subcutaneous tissue incision; \\ - Incise the cricothyroid membrane using a horizontal incision; \\ - Maintain the opening with scalpel blade turned cephalad-caudad $O R$ replace the scalpel blade \\ with your finger; \\ Place a bougie through the cricothyroid membrane and downwards into the trachea; \\ Railroad a 6.0 cuffed endotracheal tube over the bougie into the trachea; inflate cuff; \\ Confirm endotracheal tube position with waveform capnography; \\ - $\quad$ Re-oxygenate the patient, then suction the endotracheal tube for blood.
}

Figure Cannot intubate, cannot oxygenate (CICO) checklist mnemonic Aide-mémoire mnémotechnique «Impossible d'intuber, impossible d'oxygéner (CICO) »

"slow change blindness." 11 This can increase the likelihood that the clinician experiences task fixation with their initial plan (e.g., perseveration with tracheal intubation). Calling for help early, providing information, but then asking the helper to summarize the situation they observe is an excellent mitigating strategy. A newlyarrived helper will not be as prone to "slow change blindness" and by their mere arrival, may interrupt the primary clinician's inappropriate task fixation. To further help avoid task fixation, all team members must be encouraged to speak up with information (e.g., "this is the third attempt at intubation and the oxygen saturation is now 85\%") and to assist with understanding the information using "tripwire" language ${ }^{12}$ (e.g., "is this a CICO emergency?"). Team members can also assist by prompting correct action on the information (e.g., "should I get the cricothyrotomy set?"). Team members not directly involved in efforts to manage the airway may be in a better position to maintain situational awareness and "make the call" that a CICO emergency is evolving or has occurred. For this approach to be effective, regular team practice sessions are required during which all members practice speaking unambiguously while avoiding mitigating language. ${ }^{2,3}$ This includes using graded assertiveness ranging from prompts, to challenges, to instructions (e.g., from "Is this a CICO emergency?" to "This is a CICO emergency-I'm getting the cricothyrotomy equipment."). For their part, clinicians must be prepared and open to receive both information and graded assertiveness prompts.

\section{I: incision is imminent}

Once information has been obtained, and once it is understood, the clinician needs to correctly act. This means coordinating the collective team effort and performing eFONA without delay. For the CICO emergency, delay might occur because of a clinician's reluctance to clear the psychological hurdle of accepting that a rare and unexpected CICO emergency is occurring and that this in turn requires the unusual act of cutting the neck with a scalpel. Avoiding negative language may be 
beneficial. For example, rather than addressing a "failed airway" or "failed oxygenation situation", eFONA should be regarded as a positive management strategy applied to the CICO emergency to save the patient from hypoxemic brain damage or death. To this end, eFONA itself can be demystified by performing cricothyroidotomy regularly in an in vitro task trainer, ${ }^{13}$ to aid development of motor memory. Others have espoused an eFONA "priming" principle during routine airway management, whereby eFONA resources are automatically mobilized after the failure of any two of face mask or SGA oxygenation or tracheal intubation, even if a CICO emergency has not yet completely developed. ${ }^{14}$ While equipment for eFONA is obtained and opened, other issues that should be addressed at this stage include confirming pharmacologic paralysis, making a final attempt at oxygenation with face mask and SGA ventilation, and delegating the most qualified person already present to perform eFONA. The team should not delay eFONA by waiting for a more experienced person to arrive.

\section{C: cut vertically}

For the clinician who does not regularly perform surgical airway procedures, we recommend a vertical incision to initiate eFONA. The cricothyroid membrane (CTM) provides the most superficial access to the infraglottic airway. Unfortunately, the CTM is not necessarily easily identified on palpation. ${ }^{15}$ Therefore, we espouse beginning eFONA with a midline 4-6 cm vertical incision over the estimated level of the CTM and extending this incision if required. This standard approach obviates the need to decide whether to perform an initial horizontal or vertical incision based on whether the CTM is thought to be palpable. ${ }^{6,15}$ Once the vertical incision is made, the location of the CTM can be palpated within the wound, allowing for horizontal incision of the CTM to access the airway. Some bleeding should be expected.

\section{O: over-a-bougie technique}

Different methods of eFONA have been described. Unfortunately, guidance on a single preferred method is unlikely to be forthcoming from randomized controlled trials. Given recommendations of current guidelines ${ }^{6,7,16}$ and success rates in published case series, ${ }^{17,18}$ we espouse the scalpel-bougie technique in the adult patient, using a \#10 or \#20 scalpel blade, a tracheal tube introducer (bougie), and a small (e.g., \#6.0) endotracheal tube (ETT). After horizontal incision through the CTM, the scalpel blade can be rotated to a cephalad-caudad direction to maintain an opening in the cricothyroid space for subsequent bougie passage. Alternatively, a finger can be used to maintain access to the airway for bougie passage after the CTM has been incised and the scalpel removed. Either way, the ETT is then passed over the bougie, its cuff inflated within the trachea, and placement confirmed using waveform capnography (even in a newly arrested patient). ${ }^{19}$ After the patient is re-oxygenated, the ETT should be suctioned for blood.

\section{Conclusion}

Fortunately, airway management is usually accomplished without incident. Nevertheless, difficulty can be unexpected and might lead to hypoxemic brain injury or death. Our goal is to bolster patient safety and team competence by building a shared mental model. The CICO mnemonic cognitive aid may be helpful but is only as good as the team that regularly practices for this airway emergency. Importantly, we have not provided empiric evidence that the CICO cognitive aid improves communication, performance, or outcome. Nevertheless, we submit that, used as a cognitive aid (Figure), it has the potential to focus attention on the CICO emergency by providing a structured communication and action tool regardless of specialty, profession, or team member seniority. We also believe that this structured format has the potential to increase team confidence, cohesion, and performance during the rare CICO emergency.

\section{Améliorer la communication, le travail d'équipe et les actions au cours d'une urgence «Impossible d'intuber - impossible d'oxygéner (CICO)»: utilisation de l'abréviation CICO comme aide mnémotechnique}

L'anesthésiologie, les soins intensifs et la médecine d'urgence ont souvent un objectif commun: assurer une gestion sécuritaire des voies aériennes. Alors que les gestes sont réalisés le plus souvent sans incident notable, une anatomie difficile, une physiologie particulière ou l'acuité d'une situation peut précipite une urgence respiratoire. L'évolution d'une urgence des voies respiratoires peut être le résultat, aussi bien, d'habiletés non techniques que d'une expertise technique. ${ }^{1-5}$ Les lignes directrices sur les voies respiratoires insistent aujourd'hui sur l'importance de ces 
compétences non techniques, telles que le travail d'équipe et la communication efficace au cours des urgences respiratoires. ${ }^{6,7}$

Un exemple d'urgence des voies respiratoires nécessitant un travail d'équipe structuré en temps opportun est la rare situation, mettant la vie en danger, d'un patient «impossible à intuber, impossible à oxygéner » (CICO — cannot intubate, cannot oxygenate). L'urgence CICO nécessite de passer immédiatement des tentatives d'intubation trachéale à une procédure d'urgence d'accès cervical antérieur aux voies aériennes (eFONA emergency front-of-neck airway). ${ }^{6, A}$ Bien que souvent techniquement réussie, l'eFONA est pratiquée souvent trop tard pour sauver le patient de lésions cérébrales hypoxiques ou du décès. ${ }^{1,8} \mathrm{Ce}$ retard peut refléter l'absence d'habiletés non techniques vitales, c'est-à-dire un modèle mental établi et partagé sur ce qui est en train de se passer et sur le déclenchement des étapes prévisibles suivantes requises pour remédier à l'urgence CICO. ${ }^{9}$ L'absence d'un tel modèle mental partagé peut être lié: i) au fait de ne pas identifier une urgence CICO; ii) au fait, une fois l'urgence reconnue, de ne pas annoncer sans attendre et de manière non équivoque, une urgence CICO à l'ensemble de l'équipe; et/ou iii) une fois l'urgence déclarée, au faut de ne pas pratiquer rapidement une eFONA. Des aides cognitives, comme des listes de contrôle, semblent être plus efficaces si elles orientent l'action plutôt que lorsqu'elles se contentent d'énumérer des préoccupations. $^{10}$ Cet article propose une méthode mnémotechnique simple, sous forme d'aide cognitive en quatre parties, correspondant aux lettres «C-I-C-O» qui peuvent être facilement retenues et qui résument les étapes successives essentielles pour l'identification et la gestion d'une urgence respiratoire CICO.

\section{C: constater et communiquer}

Le « CICO » est défini par l'incapacité d'intuber par voie trachéale un patient (même après une seule tentative) combinée à l'échec du maintien d'une oxygénation satisfaisante en utilisant un masque facial et un dispositif de ventilation supraglottique (masque laryngé ou autre). Le retard à l'identification d'une urgence CICO en développement est multifactoriel; néanmoins, elle implique souvent une connaissance inadéquate ou une détérioration de l'appréciation de la situation. ${ }^{9}$ L'appréciation de la situation implique d'obtenir l'information (p. ex., «je ne parviens pas à intuber le patient par voie trachéale et maintenir sa saturation en oxygène malgré des tentatives optimales de ventilation au masque facial et au masque laryngé »), puis la compréhension de l'information (p. ex., « il s'agit d'une urgence $\mathrm{CICO}$ et le besoin d'une eFONA est imminent »); enfin, une action adaptée à l'information (p. ex., « il est inutile de faire de nouvelles tentatives d'intubation: une eFONA doit avoir lieu le plus rapidement possible »).

L'obtention ou la compréhension de l'information, à savoir qu'une urgence CICO se déroule, peut être occultée si l'évolution est plus progressive que soudaine, créant une « cécité aux changements lents ». ${ }^{11}$ Cela peut augmenter la probabilité que le clinicien se fixera sur la réalisation de son plan initial (c'est-à-dire en persévérant à tenter une intubation trachéale). Une excellente stratégie de limitation des risques consiste à appeler précocement de l'aide, communiquer l'information, puis demander aux personnes venant assister de résumer la situation telle qu'ils l'observent. Une aide nouvellement arrivée ne sera pas autant sujette à la «cécité aux changements lents » et sa seule arrivée peut interrompre la fixation du premier clinicien sur sa tâche inadéquate. Pour contribuer encore plus à éviter une fixation sur la tâche, tous les membres de l'équipe doivent être encouragés à parler (p. ex., « c'est la troisième tentative d'intubation et la saturation en oxygène est maintenant à $85 \% »$ ) et à aider à comprendre l'information en tendant une perche (un «fil déclencheur» $^{12}$ (p. ex. «ne s'agit-il pas d'une urgence CICO?»). Les membres de l'équipe peuvent aussi contribuer en proposant l'action correcte à mener à partir de l'information (p. ex., « dois-je aller chercher le matériel de cricothyrotomie? »). Les membres de l'équipe qui ne sont pas directement impliqués dans les efforts de gestion des voies aériennes sont peut-être dans une meilleure position pour maintenir la sensibilisation à la situation et « passer l'appel » pour indiquer qu'une urgence CICO est en train de se développer ou est déjà présente. Pour que cette démarche soit efficace, des séances régulières de pratique en équipe sont nécessaires; au cours de ces séances, tous les membres de l'équipe s'exprimeront sans ambiguité et éviteront un langage à mots couverts. ${ }^{2,3}$ Cela inclut de s'exprimer de manière affirmative et graduelle, commençant par les questions, puis passant aux problèmes, et finalement aux instructions (p. ex., de "S'agit-il d'une urgence CICO ? » à « C'est une urgence CICO » et « Je vais chercher le matériel de cricothyrotomie »). De leur côté, les cliniciens doivent être préparés et ouverts à recevoir à la fois l'information et les questions affirmatives graduées.

\section{I: L'incision est imminente}

Une fois l'information obtenue, et une fois comprise, le clinicien doit agir correctement. Cela signifie la coordination de l'effort collectif de l'équipe et la réalisation sans délai de l'eFONA. En cas d'urgence 
CICO, un retard peut survenir en raison des réticences d'un clinicien à dépasser l'obstacle psychologique que constitue l'acceptation qu'une urgence CICO, rare et inattendue, est en train de se dérouler; cela nécessite en outre l'acte inhabituel d'inciser le cou avec un bistouri. Il est sans doute préférable d'éviter d'employer des expressions négatives. Plutôt que dire, par exemple, qu'il s'agit d'un «échec des voies respiratoires» ou «d'un échec d'oxygénation », l'eFONA doit être vue comme une stratégie positive de gestion appliquée de l'urgence CICO pour protéger le patient des lésions cérébrales par hypoxie ou du décès. Dans ce but, l'eFONA peut être démystifiée en pratiquant régulièrement des cricothyroïdotomies sur un simulateur in vitro, ${ }^{13}$ pour aider à développer sa mémoire motrice. D'autres ont adopté un principe «d'amorçage » de l'eFONA au cours de la gestion des voies aériennes en routine: les ressources d'eFONA sont alors automatiquement mobilisées après l'échec d'une oxygénation au masque facial ou par masque laryngé, ou de l'intubation trachéale, même si une urgence CICO n'est pas encore complètement installée. ${ }^{14}$ Pendant que le matériel d'eFONA est apporté et préparé, d'autres problèmes doivent être résolus à ce stade, notamment la confirmation de la paralysie pharmacologique, la réalisation d'une dernière tentative de ventilation au masque facial ou laryngé, et la délégation de la réalisation de l'eFONA à la personne la plus qualifiée déjà présente. L'équipe ne doit pas retarder l'eFONA pour laisser à une personne plus expérimentée le temps d'arriver.

\section{C: Couper verticalement}

Nous recommandons à un clinicien qui ne pratique pas souvent des procédures chirurgicales sur les voies aériennes de commencer l'eFONA par une incision verticale. La membrane cricothyrö̈dienne (MCT) offre l'accès le plus superficiel aux voies aériennes infraglottiques. Malencontreusement, la MCT n'est pas toujours facile à identifier par la palpation. ${ }^{15}$ Nous sommes donc en faveur de commencer l'eFONA par une incision verticale de 4 à $6 \mathrm{~cm}$ sur la ligne médiane, au-dessus du niveau estimé de la MCT et de prolonger l'incision si nécessaire. Cette approche standard évite d'avoir à choisir entre pratiquer une incision initiale horizontale ou verticale selon que l'on estime palper la MCT. ${ }^{6,15}$ Une fois l'incision verticale pratiquée, la MCT peut être localisée par palpation dans l'incision et cette dernière peut à son tour être incisée horizontalement pour avoir accès à la trachée. Il faut s'attendre à avoir des saignements.

\section{O: technique sur bougie}

Différentes techniques d'eFONA ont été décrites. Malheureusement, il est peu probable que les essais contrôlés randomisés proposent d'emblée une méthode nettement préférable. Compte tenu des lignes directrices actuelles $^{6,7,16}$ et des taux de succès dans les séries de cas publiées, ${ }^{17,18}$ nous sommes en faveur à la technique bistouri-bougie chez le patient en utilisant une lame $\mathrm{n}^{\circ} 10$ ou $\mathrm{n}^{\circ} 20$, un introducteur de tube trachéal (une bougie), et un petit tube endotrachéal (p. ex., $n^{\circ} 6$ ). Après avoir incisé horizontalement la MCT, la lame du bistouri peut être tournée dans un sens craniocaudal pour maintenir l'ouverture d'un espace cricothyroïdien permettant le passage secondaire de la bougie. Un doigt peut aussi être utilisé pour conserver l'accès à la voie aérienne pour le passage de la bougie, une fois la MCT incisée et le bistouri éloigné. Dans un cas comme dans l'autre, le tube endotrachéal peut alors être passé par-dessus la bougie, son ballonnet est gonflé dans la trachée et son positionnement est confirmé par le tracé de la capnographie (même chez un patient en arrêt récent). ${ }^{19}$ Le patient peut enfin être réoxygéné et le sang présent dans le tube endotrachéal est aspiré.

\section{Conclusion}

Heureusement, la gestion des voies aériennes est habituellement menée sans incident. Des difficultés peuvent toutefois survenir de manière inattendue et déboucher sur des lésions du cerveau par hypoxie ou sur un décès. Notre objectif est de renforcer la sécurité des patients et la compétence de l'équipe en créant un modèle mental partagé. La méthode mnémotechnique cognitive de la CICO peut être utile, mais ne l'est que dans la mesure où l'équipe s'est régulièrement entraînée à faire face à cette urgence des voies aériennes. Il faut rappeler que nous n'avons pas fourni de preuves empiriques que l'aide cognitive « CICO » améliore la communication, la performance ou les résultats. Nous pensons néanmoins qu'utilisée comme aide cognitive, elle peut aider à porter une plus grande attention à l'urgence CICO en proposant une communication structurée et un outil pour agir indépendamment de la spécialité, de la profession ou de l'ancienneté du membre de l'équipe. Nous pensons également que ce format structuré peut accroître la confiance, la cohésion et la performance de l'équipe au cours de la rare urgence d'une CICO.

Conflicts of interest None declared. 
Editorial responsibility This submission was handled by Dr. Hilary P. Grocott, Editor-in-Chief, Canadian Journal of Anesthesia.

Conflits d'intérêt Aucun déclaré.

Responsabilité éditoriale Cet article a été traité par le $\mathrm{D}^{\mathrm{r}}$ Hilary $\mathrm{P}$. Grocott, Rédacteur en chef, Journal canadien d'anesthésie.

\section{References}

1. The Royal College of Anaesthetists; The Difficult Airway Society. 4th National Audit Project (NAP 4). Major complications of airway management in the UK. Report and Findings, March 2011. The Royal College of Anaesthetists, London, 2011. Available from URL: https://rcoa.ac.uk/nap4 (accessed July 2018).

2. St Pierre $M$, Hofinger $G$, Buerschaper $C$, Simon $R$. Crisis Management in Acute Care Settings. 2nd ed. NY: Springer; 2011.

3. Brindley $P G$, Cardinal $P$. Optimizing Crisis Resource Management to Improve Patient Safety and Team Performance: A handbook for Acute Care Health Professionals. Royal College of Physicians and Surgeons Press; 2017. Available from URL. https:// psnet.ahrq.gov/resources/resource/31405/optimizing-crisis-resour ce-management-to-improve-patient-safety-and-team-performance-ahandbook-for-acute-care-health-professionals. (accessed July 2018).

4. Brindley $P G$, Beed $M$, Law JA, et al. Airway management outside of the operating room: how to better prepare. Can J Anesth 2017; 64: 530-9.

5. Brindley $P G$, Beed $M$, Duggan $L V$, Hung $O$, Murphy $M F$. Updating our approach to the difficult and failed airway: time to "stop and think". Can J Anesth 2016; 63: 373-81.

6. Frerk C, Mitchell VS, McNarry AF, et al. Difficult Airway Society 2015 guidelines for management of unanticipated difficult intubation in adults. Br J Anaesth 2015; 115: 827-48.

7. Higgs A, McGrath BA, Goddard C, et al. Guidelines for the management of tracheal intubation in critically ill adults. Br J Anaesth 2018; 120: 323-52.

8. Peterson GN, Domino KB, Caplan RA, Posner KL, Lee LA, Cheney $F W$. Management of the difficult airway: a closed claims analysis. Anesthesiology 2005; 103: 33-9.
9. Flin R, Fioratou R, Frerk C, Trotter C, Cook TM. Human factors in the development of complications of airway management: preliminary evaluation of an interview tool. Anaesthesia 2013; 68: $817-25$

10. Gawande A. The Checklist Manifesto: How to Get Things Right. NY: Metropolitan Books; 2009.

11. O'Regan $J K$. Thoughts on change blindness. In: Jenkin M, Harris LR, editors. Vision and Attention. NY: Springer; 2001. p. 281302.

12. Oxford Dictionary. Available from URL: https://en. oxforddictionaries.com/definition/tripwire (accessed July 2018).

13. Duggan LV, Lockhart SL, Romano KR, Weingart SD, Levitan $R M$, Brindley $P G$. Front-of-neck airway meets front-of-neck simulation: improving cricothyrotomy skills using a novel openaccess three-dimensional model and the Airway App. Can J Anesth 2017; 64: 1079-81.

14. Australian and New Zealand College of Anaesthetists (ANZCA). Guidelines for the Management of Evolving Airway Obstruction: Transition to the Can't Intubate Can't Oxygenate Airway Emergency - 2017. Available from URL: http://www.anzca.edu. au/documents/ps61bp-2017.pdf (accessed July 2018).

15. Law JA. Deficiencies in locating the cricothyroid membrane by palpation: we can't and the surgeons can't, so what now for the emergency surgical airway? Can J Anesth 2016; 63: 791-6.

16. Law JA, Broemling N, Cooper RM, et al. The difficult airway with recommendations for management - part 1- difficult tracheal intubation encountered in an unconscious/induced patient. Can J Anesth 2013; 60: 1089-118.

17. Lockey D, Crewdson K, Weaver A, Davies G. Observational study of the success rates of intubation and failed intubation airway rescue techniques in 7256 attempted intubations of trauma patients by pre-hospital physicians. Br J Anaesth 2014; 113: $220-5$.

18. Duggan LV, Lockhart SL, Cook TM, O'Sullivan EP, Dare T, Baker PA. The Airway App: exploring the role of smartphone technology to capture emergency front-of-neck airway experiences internationally. Anaesthesia 2018; 73: 703-10.

19. Levine RL, Wayne MA, Miller CC. End-tidal carbon dioxide and outcome in out-of-hospital cardiac arrest. N Engl J Med 1997; 337: 301-6. 